\title{
El papel de los rendimientos y la diferenciación de productos como determinantes de la estabilidad del cártel*
}

\author{
Roberto Contreras Marín \\ Departamento de Economía, Universidad Tecnológica Metropolitana, \\ FAE, Santiago, Chile \\ Nikolaos Georgantzís \\ Departamento de Teoría e Historia Económica, Universidad de Granada \\ Miguel Ginés Vilar \\ Departamento de Economía y LEE, Universitat Jaume I,
}

\section{Resumen}

Estudiamos las implicaciones que tienen sobre la estabilidad del cártel, los rendimientos de la producción en presencia de productos diferenciados, cuando las empresas compiten en cantidades. Mostramos que el cártel puede ser igualmente estable en presencia de un menor grado de diferenciación siempre que las deseconomías de escala sean mayores. Además demostramos que a partir de un determinado factor de descuento pueden existir tecnologías con rendimientos de escala constantes o decrecientes donde el cártel siempre es estable independiente del grado de diferenciación.

Palabras clave: colusión, diferenciación de productos, competencia à la Cournot, rendimientos de escala.

Clasificación JEL: C70, D21, D43.

\begin{abstract}
We study cartel stability in a differentiated quantity-setting duopoly with decreasing returns to scale. We show that a cartel may be equally stable in the presence of lower differentiation, provided that the decreasing returns parameter is higher. Furthermore, we show that, above a given discount rate, a cartel may be stable for any degree of product differentiation.
\end{abstract}

Keywords: colusiun produc, product differentiation, cournot competition, scale economies.

JEL classification: C70, D21, D43.

* Deseamos agradecer a los participantes de las XXII Jornadas de Economía Industrial (IESE de Navarra, septiembre de 2006) por sus comentarios. Los errores u omisiones son exclusiva responsabilidad nuestra. Contreras desea agradecer la financiación recibida por la Universidad Tecnológica Metropolitana (Proyecto N²87/2007, Resolución N04844 Exenta). Georgantzís agradece la hospitalidad del Departamento de Economía de la Universidad de Chipre y la financiación recibida por el Ministerio de Educación y Ciencia (SEJ 2005-07544/ECON y PR 2007-0153). 


\section{Introducción}

Durante las dos últimas décadas se ha dado una gran atención al problema del mantenimiento de la estabilidad del cártel utilizando para este análisis básicamente dos modelos: el modelo no espacial de Chamberlin (1951) y el modelo espacial de Hotelling (1929) y Salop (1979).

Los trabajos que se han dedicado a estudiar la estabilidad de un cártel en un contexto espacial como no espacial, con productos diferenciados, cuando las empresas compiten en precios generalmente predicen que la diferenciación facilita la colusión. Ejemplo de ello son los trabajos de Chang (1991) y (1992), Häckner (1995) y (1996) y Rothschild (1992). Alternativamente, se encuentra que los efectos de la diferenciación sobre la sostenibilidad del cártel son ambiguos, con relaciones no monótonas entre la facilidad para coludir y el parámetro diferenciador. Ejemplo de ello son los trabajos de Ross (1992) y Rothschild (1997).

Por otra parte Rothschild (1992) muestra que, cuando la variable estratégica es la cantidad, el cártel es más estable mientras más homogéneos son los productos.

Se debe tener en cuenta que, en todos estos trabajos, los autores han considerado que los rendimientos de la producción son constantes.

Otra preocupación recurrente de la literatura se relaciona con determinar, en un superjuego con productos diferenciados, qué variable estratégica eligen las empresas para competir: precio o cantidad. Deneckere (1983) y Singh y Vives ${ }^{1}$ (1984) encuentran que, un cártel es más estable si se decide sobre cantidades cuando los bienes son sustitutos y sobre precios cuando los bienes son complementos. Por otra parte, Lambertini y Schultz (2003) encuentran que, para factores de descuento muy altos, la elección de la variable estratégica no importa. En ambos casos se obtienen los beneficios de monopolio. En cambio para factores de descuento bajo el resultado es similar a los trabajos de (1983) y (1984).

En nuestro análisis incorporamos los efectos de los rendimientos de escala. Desde esa perspectiva, Tirole (1992) menciona que los rendimientos decrecientes a escala hacen que las rebajas en los precios hoy sean menos rentables. Sin embargo, debilitan también la fuerza de futuras represalias, ya que limita la cantidad que las empresas pueden ofrecer en el mercado. Esto implica, a priori, que el efecto de los rendimientos decrecientes a escala son ambiguos ${ }^{2}$. En este mismo contexto, Lambertini y Sasaki (2001) demuestran de forma intuitiva y matemáticamente informal que, en un juego repetido de un oligopolio con altos costes marginales, hay más sitio para la colusión tácita que sus contrapartes idénticas de costes marginales más bajos, debido a la restricción del precio positivo.

${ }^{1}$ Se menciona en los trabajos realizados por LAMBERTINI y SCHULTZ (2002) y por HÄCKNER (2000).

2 Para este comentario Tirole hace referencia al trabajo realizado por BROCK y SCHEINKMAN (1985). 
Finalmente debemos mencionar que los resultados obtenidos por los estudios empíricos contrastan con los teóricos. Así Hay y Kelley (1974) encuentran que es más probable que la colusión ocurra y perdure cuando: el número de miembros del cártel es pequeño, la concentración es alta y los productos son homogéneos. A su vez Symeonidis (2003) encuentra que, industrias con publicidad intensiva son también industrias con productos diferenciados, y la coordinación sobre un precio colusivo o un conjunto de precios colusivos se dificulta por la diferenciación de productos, debido a la incertidumbre sobre las características del producto rival o por la necesidad de renegociar frecuentemente los siguientes cambios en esas características.

Desde esa perspectiva, surge el interés de estudiar los efectos que tienen sobre la estabilidad del cártel, en un juego repetido de un duopolio con ajuste en cantidad ${ }^{3}$, los rendimientos de escala con productos diferenciados, evaluando la interacción conjunta de estos dos parámetros. Así, deseamos observar si se alteran los resultados de los modelos que consideran rendimientos de escala constantes. Además, pretendemos estudiar la relación que se obtiene entre el parámetro diferenciador y el parámetro de los rendimientos de escala para un mismo valor del factor de descuento crítico.

\section{Descripción del modelo}

Utilizaremos un modelo simétrico no espacial o modelo de Chamberlin, similar al empleado por Lambertini y Schultz (2003) con una excepción: la producción por parte de las empresas tendrá unos costes $\left(c_{i}\right)$ que serán función no lineal del output de cada una de las empresas.

Considere una industria compuesta por dos firmas $i$ y $j$. Cada firma produce una variedad de un producto diferenciado que se enfrenta a la siguiente función inversa de la demanda:

$$
p_{i}=1-q_{i}-\gamma q_{j}
$$

Así $q_{i}, p_{i}$ representan respectivamente las cantidades y los precios de cada una de las firmas. Por otra parte, $\gamma$ sirve como una medida del grado de diferenciación entre los productos, si $\gamma$ es uno, entonces, los bienes son sustitutos perfectos. Por el contrario, si $\gamma$ es cero, entonces son bienes con funciones de demanda independientes. Por tanto los bienes son sustitutos imperfectos ${ }^{4}$ si $0 \leq \gamma<1$. Así la función de costes de la firma viene representada por:

$$
\boldsymbol{c}_{\boldsymbol{i}}=c q_{i}+\frac{1}{2} d q_{i}^{2}
$$

${ }^{3}$ En CONTRERAS y otros (2008) planteamos el mismo objetivo en el contexto de un duopolio $\grave{a}$ la Bertrand.

4 También se puede indicar que los bienes son complementarios, si $-1<g \leq 0$. Pero nosotros descartamos este caso. 
Para que los costes, los precios, las cantidades y los beneficios sean positivos, suponemos ${ }^{5}$ que:

$$
d \geq 0 \text { y } c \in[0,1]
$$

Como $d$ es mayor o igual a cero, nos encontramos en la zona donde los rendimientos de la producción son constantes o decrecientes.

Por otra parte, siguiendo la terminología habitual, 1-c puede considerarse como el tamaño de mercado.

\subsection{Cooperación en cantidades (equilibrio colusivo) en un período}

En nuestro modelo la solución cooperativa para las empresas involucra la maximización de los beneficios conjuntos respecto a las cantidades. De ellas se obtienen las condiciones de primer orden y de la solución ${ }^{6}$ del sistema se obtienen las cantidades de equilibrio a partir de las cuales obtendremos los beneficios colusivos de cada una de las empresas, esto es:

$$
\begin{aligned}
& \pi_{1}+\pi_{2}=p_{1} q_{1}-c_{1}+p_{2} q_{2}-c_{2} \Rightarrow \underbrace{\frac{\partial\left(\pi_{1}+\pi_{2}\right)}{\partial q_{1}}=0 \wedge \frac{\partial\left(\pi_{1}+\pi_{2}\right)}{\partial q_{2}}=0}_{\text {Condiciones de primer orden }} \\
& \underbrace{q_{1}}_{q_{i c}=\frac{1-c-2 \gamma q_{2}}{2+d} \wedge q_{2}=\frac{1-c-2 \gamma q_{1}}{2+d}} \Rightarrow \\
& \frac{1-c}{2+d+2 \gamma} \Rightarrow p_{i c}=\frac{(1+\gamma)(1+c)+d}{2+d+2 \gamma} \Rightarrow \pi_{i c}=\frac{(1-c)^{2}}{2(2+d+2 \gamma)}
\end{aligned}
$$

${ }^{5}$ Aunque nuestro análisis se pude extender al caso de rendimientos crecientes, nos concentramos en el caso de rendimientos constantes y decrecientes para evitar complicaciones innecesarias, relacionadas con la existencia de equilibrio.

${ }^{6}$ En el caso de un cártel bajo (rendimientos crecientes), sería mejor producirlo todo en una empresa, dejándose de producir una de las dos variedades. Y es este mismo hecho (los productos son diferenciados y no homogéneos como en ROTHSCHILD (1999)) que hace que tal reparto de la producción se haga imposible por ser ilegal, en un contexto en el que la colusión explícita está perseguida por la Ley. Por eso asumimos que la desaparición total de una de las dos variedades y la consiguiente solución asimétrica en el caso del cártel bajo rendimientos crecientes no es posible por razones institucionales. Así nos seguiremos centrando en el caso simétrico (pactos sobre restricción, pero no desaparición o reparto productivo de uno de los dos output). 


\subsection{Competencia en cantidades (equilibrio Cournot-Nash)}

El proceso es similar al anterior, pero viene de la solución simultánea de la maximización de los beneficios individuales, esto es:

$$
\begin{aligned}
& \pi_{i}=p_{i} q_{i}-c_{i} \Rightarrow \underbrace{\frac{\partial \pi_{i}}{\partial q_{i}}=0}_{\text {Condiciones de primer orden }} \Rightarrow \underbrace{q_{1}=\frac{1-c-\gamma q_{2}}{2+d} \wedge q_{2}=\frac{1-c-\gamma q_{1}}{2+d}}_{\text {Funciones dereacción }} \Rightarrow \\
& q_{\text {in }}=\frac{1-c}{2+d+\gamma} \Rightarrow p_{\text {in }}=\frac{1+d+c(1+\gamma)}{2+d+\gamma} \Rightarrow \pi_{\text {in }}=\frac{(1-c)^{2}(2+d)}{2(2+d+\gamma)^{2}}
\end{aligned}
$$

\subsection{Desviación unilateral de la cantidad pactada (una empresa hace trampas)}

Una de las empresas determina la cantidad y fija la desviación óptima del acuerdo colusivo, mientras la otra empresa respeta el acuerdo (empresa engañada), suponiendo inicialmente que es la empresa 1 la que se desvía del acuerdo colusivo y la empresa 2 respeta el acuerdo ${ }^{7}$.

$$
\begin{aligned}
& q_{2 c}=\frac{1-c}{2+d+2 \gamma} \Rightarrow \pi_{1}=-c q_{1}+\left(1-\frac{(1-c) \gamma}{(2+d+2 \gamma)}-q_{1}\right) q_{1}-\frac{d q_{1}^{2}}{2} \Rightarrow \frac{\partial \pi_{1}}{\partial q_{1}}=0 \Rightarrow \\
& q_{i d}=\frac{(1-c)(2+d+\gamma)}{(2+d)(2+d+2 \gamma)} \Rightarrow p_{i d}=\frac{(1+d)(2+d+\gamma)+c(2+d+\gamma(3+d))}{(2+d)(2+d+2 \gamma)} \Rightarrow \pi_{i d}=\frac{(1-c)^{2}(2+d+\gamma)^{2}}{2(2+d)(2+d+2 \gamma)^{2}} \Rightarrow \\
& p_{i e}=\frac{2+d^{2}+2 \gamma-\gamma^{2}+d(3+\gamma)+c\left(2+2 \gamma+\gamma^{2}+d(1+\gamma)\right)}{(2+d)(2+d+2 \gamma)} \Rightarrow \\
& \pi_{i e}=\frac{(1-c)^{2}\left[4+d^{2}+4 \gamma-2 \gamma^{2}+2 d(2+\gamma)\right]}{2(2+d)(2+d+2 \gamma)^{2}}
\end{aligned}
$$

\subsection{Estructura de pagos del juego}

Representamos la matriz de pagos de este juego, después de dividir el resultado por $\frac{\left(1-c^{2}\right)}{2}$ con el propósito de simplificar los resultados y operar con más facilidad:

\footnotetext{
${ }^{7}$ El resultado es simétrico cuando se plantea la situación inversa.
} 
Matriz de pagos dividida por $(1-c)^{2} / 2$

\begin{tabular}{|c|c|c|c|c|}
\hline & \multicolumn{4}{|c|}{ Empresa 2} \\
\hline \multirow{4}{*}{$\overline{\widetilde{R}}$} & & & espetar & No respetar \\
\hline & \multirow{2}{*}{ Respetar } & 1 & 1 & $\left(4+d^{2}+4 \gamma-2 \gamma^{2}+2 d(2+\gamma)\right] \quad(2+d+\gamma)^{2}$ \\
\hline & & $(2+d+2$ & $\left.2 \gamma)^{\prime}(2+d+2 \gamma)\right)$ & $(2+d)(2+d+2 \gamma)^{2}, \overline{(2+d)(2+d+2 \gamma)^{2}}$ \\
\hline & $\begin{array}{c}\text { No } \\
\text { respetar }\end{array}$ & $\left(\frac{(2+d+\gamma)^{2}}{(2+d)(2+d+2 \gamma)^{2}}\right.$ & $\frac{\left[4+d^{2}+4 \gamma-2 \gamma^{2}+2 d(2+\gamma)\right]}{(2+d)(2+d+2 \gamma)^{2}}$ & $\left(\frac{(2+d)}{(2+d+\gamma)^{2}}, \frac{(2+d)}{(2+d+\gamma)^{2}}\right)$ \\
\hline
\end{tabular}

\section{Colusión en un juego repetido}

La política de la competencia de las economías modernas impide celebrar contratos legales sobre acuerdo colusivos. Por tanto, la colusión debe ser equilibrio de Nash perfecto en subjuegos (NPS) de un juego repetido infinitas veces, para que sea sostenible. En general, la colusión puede sostenerse, si los beneficios descontados de respetar el acuerdo colusivo son mayores o iguales que, los beneficios descontados de jugar el equilibrio no cooperativo de Cournot.

La estrategia colusiva que utilizaremos en nuestro modelo es la de Friedman (1971) quien define una estrategia «tipo gatillo» para un juego repetido.

Definiremos $\pi_{i c}$ como el beneficio colusivo de la empresa $i$, si las empresas adhieren a la estrategia colusiva. En el caso de una empresa que decide engañar a sus socios de cártel, $\pi_{i d}$ es el beneficio de la empresa $i$ que se desvía de la estrategia colusiva. Y $\pi_{i n}$ es el beneficio que la empresa $i$ gana en el equilibrio no cooperativo de Cournot.

\section{Factor de descuento crítico}

El factor de descuento critico $\left(\alpha^{*}\right)$ que utilizaremos, es similar al utilizado por Rothschild (1997) y Martin (1993). Así coludir será una opción factible si, el factor de descuento $(\alpha)$ es mayor o igual que el factor de descuento crítico $\left(\alpha^{*}\right)$. En nuestro caso, si:

$$
\alpha \geq=\alpha^{*}=\frac{\pi_{i d}-\pi_{i c}}{\pi_{i d}-\pi_{i n}}=\frac{1}{2}+\frac{\gamma^{2}}{\left.2\left[2(2+d+\gamma)^{2}-\gamma^{2}\right)\right]}
$$

En adelante utilizaremos el factor de descuento crítico como medida de la estabilidad del cártel. Entonces, para valores relativamente altos del factor de descuen- 
to crítico (cercanos a 1) el cártel es más difícil de sostener, por el contrario, para valores bajos del factor de descuento crítico (cercanos a 0.5 ) el cártel es más fácil de sostener.

\section{Resultados}

A partir de la expresión anterior encontramos una relación lineal y positiva entre los rendimientos de la producción y la diferenciación de productos para un determinado valor del factor de descuento crítico. Esto es:

$$
d=-2+\left(\sqrt{\frac{\alpha^{*}}{2 \alpha^{*}-1}}-1\right) * \gamma
$$

Lo anterior nos conduce a la siguiente proposición:

Proposición 1: Para un determinado valor constante del factor de descuento crítico y cuando d es mayor o igual a cero, podremos encontrar industrias que coluden con un producto más homogéneo que otras, siempre que, los rendimientos de la producción también sean más decrecientes o las deseconomías de escala sean más intensas.

Por lo tanto, podemos indicar que existe un grado de sustitubilidad constante de $\left(\sqrt{\left(\alpha^{*} /\left(2 \alpha^{*}-1\right)\right)}-1\right)$ entre los rendimientos de la producción $(d)$ y la diferenciaación de productos $(\gamma)$ para que un cártel pueda ser igualmente fácil de sostener.

La relación que existe entre los rendimientos de la producción y la diferenciación de productos se puede apreciar en la figura 1-A. En ella se puede constatar que, dado un determinado factor de descuento crítico constante a partir del cual es posible mantener la colusión, se podrá encontrar industrias con un menor o mayor grado de diferenciación dependiendo de los rendimientos de la producción que estas tengan. Así, mientras más decrecientes sean los rendimientos de la producción, más homogéneos podrán ser los productos. Además podemos indicar que la pendiente de la recta que relaciona la diferenciación de los productos con los rendimientos de la producción decrece a medida que el factor de descuento crítico $\left(\alpha^{*}\right)$ se incrementa, esto nos indica que, cuando el factor de descuento crítico aumenta, ante una variación del grado de homogeneidad de los productos, se necesitará una variación cada vez menor de los rendimientos de la producción para poder sostener la colusión.

En la figura 1-B se puede apreciar que todas las tecnologías que se encuentran sobre o encima de la recta tienen un factor de descuento crítico menor o igual que el factor de descuento que para este ejemplo en particular es de 0.51 y por tanto, estos cárteles son sostenibles. 
En la figura 1-C, el factor de descuento se encuentra siempre por debajo de la zona en que los rendimientos de la producción son constantes o decrecientes. Así, para este caso en particular, se podrá coludir independientemente del grado de diferenciación o de homogeneidad que tengan los productos.

En general, se puede hacer un planteamiento en el que para un determinado valor del factor de descuento, siempre será posible encontrar unos rendimientos de la producción a partir de los cuales el factor de descuento crítico sea menor o igual que el factor de descuento y, por tanto, el cártel será estable independiente del grado de homogeneidad o diferenciación.

Corolario 1: Cuando el factor de descuento $(\alpha)$ es mayor que 0,5294, todas las industrias (o duopolios) con rendimientos constantes o cualquier tipo de rendimientos decrecientes y cualquier grado de diferenciación del producto pueden ser sostenibles como cártel.

FIGURA 1

RELACIÓN ENTRE LOS RENDIMIENTOS DE LA PRODUCCIÓN Y EL PARÁMETROS DIFERENCIADOR PARA DISTINTOS VALORES DEL FACTOR DE DESCUENTO CRÍTICO $(\alpha *)$

FIGURA 1-A

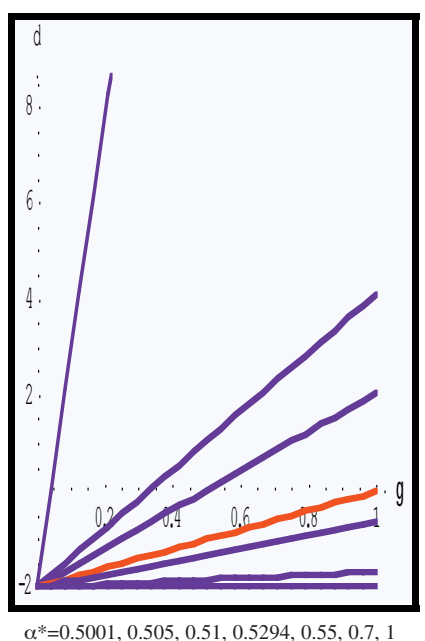

FIGURA 1-B

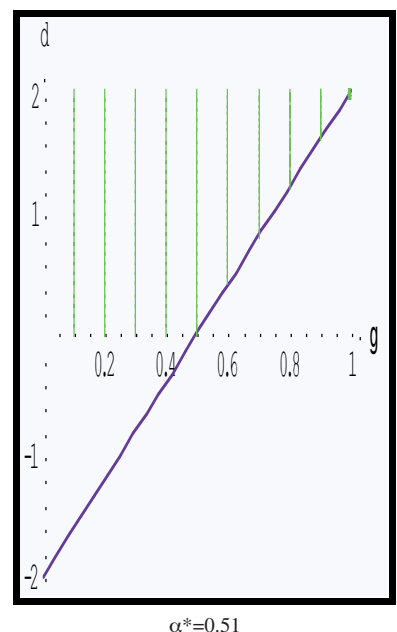

FIGURA 1-C

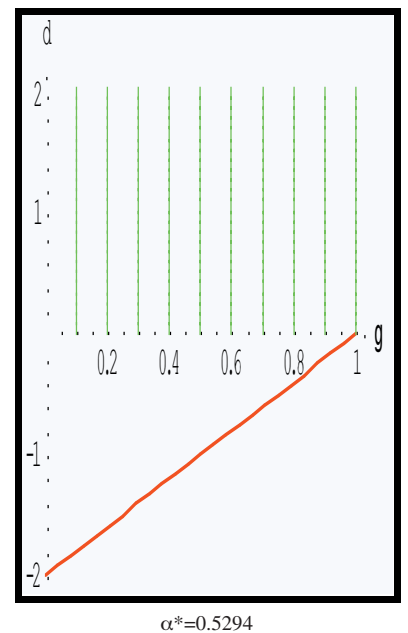

Otra de nuestras preocupaciones es poder determinar qué ocurre con el factor de descuento crítico necesario para sostener la colusión con un grado de diferenciación constante a medida que los rendimientos de la producción se hacen más decrecientes.

$$
\frac{\partial \alpha^{*}}{\partial d}=-\frac{2 \gamma^{2}(2+d+\gamma)}{\left(8+2 d^{2}+8 \gamma+\gamma^{2}+4 d(2+\gamma)\right)^{2}}
$$

Se puede apreciar que existe una variación negativa entre los rendimientos de la producción y el factor de descuento crítico necesario para sostener la colusión. 
Corolario 2: A medida que las deseconomías de escala son más intensas, el factor de descuento crítico necesario para sostener la colusión disminuye.

Para empresas con un determinado grado de diferenciación constante, encontramos que, mientras más decrecientes son los rendimientos de la producción, más fácil es sostener la colusión, esto se puede apreciar en la figura 2, donde cada una de las curvas representan la variación del factor de descuento crítico necesaria para que, incrementando el parámetro $d$, se pueda mantener un determinado nivel de diferenciación. Cuanto más alejada del origen se encuentre la curva, más homogéneos son los productos. Así, podemos observar que, mientras más homogéneos son los productos, más decrecientes deben ser los rendimientos de la producción para que el factor de descuento crítico sea menor.

\section{FIGURA 2}

\section{LOS RENDIMENTOS DE LA PRODUCCIÓN Y EL FACTOR DE DESCUENTO CRÍTICO}

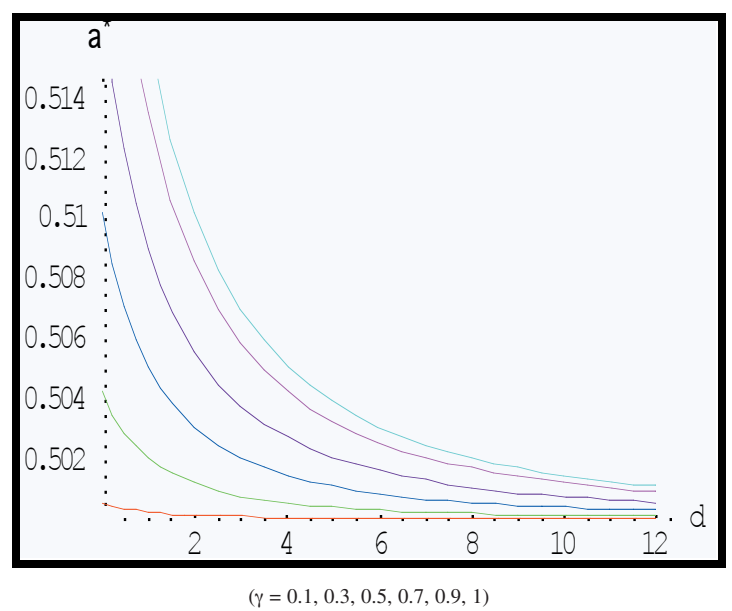

Por otra parte, qué ocurre con el factor de descuento crítico necesario para sostener la colusión con unos determinados rendimientos de la producción a medida que incrementamos la homogeneidad de los productos.

$$
\frac{\partial \alpha^{*}}{\partial \gamma}=\frac{2(2+d) \gamma(2+d+\gamma)}{\left(8+2 d^{2}+8 \gamma+\gamma^{2}+4 d(2+\gamma)\right)^{2}}
$$

Se puede apreciar que existe una variación positiva entre el grado de homogeneidad de los productos y el factor de descuento crítico necesario para sostener la colusión. Así podemos llegar al siguiente Corolario. 
Corolario 3: Para empresas con unos determinados rendimientos de producción fijos, encontramos que, mientras más diferenciados son los productos, más fácil es sostener la colusión.

Así, mientras más homogéneos son los productos, mayor será el valor del factor de descuento crítico $\left(\alpha^{*}\right)$ necesario para mantener la colusión. Esto implica que más difícil es sostener la colusión. Esto contrasta con lo descrito por Rothschild (1992) para el análisis del factor de descuento cuando se compite en cantidades y concuerda con el análisis de éste autor cuando se compite en precios. En ambos casos, los costes marginales son constantes y se utiliza el modelo de Chamberlin. También concuerda con los trabajos de Chang (1991), Ross (1992) y Häckner (1996), en donde se analiza la facilidad para coludir, utilizando un modelo espacial de competencia en precios.

Finalmente nos interesa ver qué ocurre con el factor de descuento crítico necesario para sostener la colusión cuando variamos los rendimientos de la producción y el grado de diferenciación de los productos.

$$
\frac{\partial^{2} \alpha^{*}}{\partial d \partial \gamma}=-\frac{2 \gamma\left[-2 \gamma(2+d+\gamma)^{2}+\gamma(4+2 d+\gamma)+4(2+d)(2+d+\gamma)^{2}\right]}{\left[-\gamma^{2}+2(2+d+\gamma)^{2}\right]^{3}}
$$

Proposición 2: Dado $d_{1}<d_{2}$, encontramos que $\frac{\partial \alpha}{\partial \gamma}\left|d_{1}>\frac{\partial \alpha}{\partial \gamma}\right| d_{2}$. Así, cuanto memenores son los rendimientos de la producción o menores sean las deseconomías de escala mayor es el esfuerzo necesario en diferenciación de producto para sostener la colusión.

Así, a medida que se incrementan las deseconomías de escala o los rendimientos de escala se hacen más decrecientes, la variación del factor de descuento crítico respecto a la homogeneidad de los productos disminuye y, por tanto, se incrementa la estabilidad del cártel. Por el contrario, cuanto menor es $d$, la variación del factor de descuento crítico respecto a la homogeneidad de los productos se incrementa y, por consiguiente, más fácil será sostener la colusión.

Como anticipáramos existe una evidente interacción entre los rendimientos de la producción y el grado de diferenciación de los productos en la facilidad para coludir, esto significa que se mueven en dirección contraria, pudiendo contrarrestarse los efectos de uno de ellos respecto al otro en la facilidad para coludir.

\section{Conclusiones}

Como anticipamos existe una interacción entre los rendimientos de la producción y el grado de diferenciación de los productos en la facilidad para coludir. Así, encon- 
tramos que existe una relación lineal y directa entre los rendimientos de la producción y el grado de homogeneidad de los productos para un determinado valor del factor de descuento crítico. Desde esa perspectiva, se podrá encontrar industrias que teniendo la misma dificultad para coludir lo podrán hacer con un producto más homogéneo que otras, siempre que, los rendimientos de la producción sean más decrecientes o las deseconomías de escala sean más intensas.

Se demuestra que, para un determinado valor del factor de descuento, siempre será posible encontrar unos rendimientos de la producción a partir de los cuales el cártel será estable independiente del grado de homogeneidad o diferenciación que exista entre los productos.

También hemos encontrado que, los rendimientos de la producción juegan un papel crucial en la facilidad para coludir. Así, para un determinado nivel de diferenciación, mientras más decrecientes sean los rendimientos de la producción, menor será el factor de descuento crítico y, por tanto, más fácil será sostener la colusión.

Finalmente podemos indicar que, nuestro trabajo puede explicar las diferencias que existen entre los estudios teóricos, que en general concluyen que mientras más diferenciados son los productos más fácil es sostener la colusión, por una parte y por otra parte los estudios empíricos, que concuerdan en que mientras más homogéneos son los productos, más fácil es sostener la colusión. Nosotros hemos demostrado que ambas situaciones son posibles dependiendo de los rendimientos de la producción que tengan las empresas.

\section{Referencias}

[1] ABREU, D. (1986). «External Equilibria of Oligopolistic Supergames». Journal of Economic Theory, 39: 191-225.

[2] BROCK, W. y SCHEINKMAN, J. (1985). «Price Setting Supergames with Capacity Constraints». Review of Economic Studies, 52: 371-382.

[3] CHAMBERLIN, E. (1951). «Monopolistic Competition Revisited». Economica, New Series, XVIII, November, 343-362.

[4] CHANG, M.-H. (1991). «The Effects of Product Diferentiation on Collusive Pricing». International Journal of Industrial Organization, 3: 453-470.

[5] CHANG, M.-H. (1992). «Intertemporal Produc Choice and Its Effects on Collusive Firm Behavior». International Economic Review, 4: 773-793.

[6] CONTRERAS, R., N. GEORGANTZIS y M. GINÉS (2008). «Colusión en un duopolio de Bertrand con rendimientos decrecientes y productos diferenciados». Estudios de Economía, 35: 19-32.

[7] DENECKERE, R (1983). «Duopoly Supergames with Product Differentiation». Economics Letters, 11: 37-42.

[8] FRIEDMAN, J.W. (1971). «A Noncooperative Equilibrium for Supergames». Review of Economic Studies, 38: 1-12.

[9] HÄCKNER, J. (1995). «Endogenous Product Design in an Infinitely Repeated Game». International Journal of Industrial Organization, 13: 277-299. 
[10] HÄCKNER, J. (1996). «Optional Symmetric Punishments in a Bertrand Diferentiated Products Duopoly». International Journal of Industrial Organization, 14: 611-630.

[11] HÄCKNER, J. (2000). «A Note on Price and Quantity Competition in Differentiated Oligopolies». Journal of Economic Theory, 93: 233-239.

[12] HAY, G. y KELLEY, D. (1974). «An Empirical Survey of Price Fixing Conspiracies». Journal of Law and Economics, 17: 13-38.

[13] HENDEL, I. y FIGUEIREDO, J.N. (1997). «Product Differentiation and Endogenous Discounting». International Journal of Industrial Organization, 16: 63-79.

[14] HOTELLING, H. (1929). «Stability in Competition». Economic Journal, 39: 41-57.

[15] LAMBERTINI, L., PODDAR, S. y SASAKI, D. (1998). «Standardization and the Stability of Collusion». Economics Letters, 58: 303-310.

[16] LAMBERTINI, L. y SASAKI, D. (2001). «Marginal Costs and Collusive Sustainability». Economics Letters, 72: 117-120.

[17] LAMBERTINI, L. y SCHULTZ, C. (2003). «Price or Quantity in Tacit Collusion?». Economics Letters, 78: 131-137.

[18] LANCASTER, K. (1966). «A New Approach to Consumer Theory». Journal of Political Economy, 74: 132-57.

[19] MARTIN, S. (1993). Advanced Industrial Economics. Oxford, UK Cambridge, Mass, USA Blackwell.

[20] ROSS, T. (1992). «Cartel Stability and Product Differentiation». International Journal of Industrial Organization, 10: 1-13.

[21] ROTHSCHILD, R. (1992). «On the Sustainability of Collusion in Differentiated Duopolies». Economics Letters, 40: 33-37.

[22] ROTHSCHILD, R. (1997). «Product Differentiation and Cartel Stability: Chamberlin versus Hotelling». Annals of Regional Science, 31: 259-271.

[23] SALOP, S. (1979). «Monopolistic Competition with Outside Goods». Bell Journal of Economics, 10: 141-156.

[24] SYMEONIDIS, G. (2003). «In which Industries Is Collusion More Likely? Evidence from the UK». The Journal of Industrial Economics, 1: 45-74.

[25] TIROLE, J. (1992). The Theory of Industrial Organization. MIT Press. 